\title{
Robustness analysis of metabolic predictions in algal microbial communities based on different annotation pipelines
}

\author{
Elham Karimi ${ }^{\text {Corresp., } 1}$, Enora Geslain ${ }^{1,2}$, Arnaud Belcour $^{3}$, Clémence Frioux $^{4}$, Méziane Aïte $^{3}$, Anne Siegel $^{3}$, Erwan \\ Corre $^{2}$, Simon M. Dittami ${ }^{\text {Corresp. } 1}$ \\ 1 UMR8227, Integrative Biology of Marine Models, Sorbonne Université/CNRS, Station Biologique de Roscoff,, Roscoff, France \\ 2 FR2424, Sorbonne Université/CNRS, Station Biologique de Roscoff, Roscoff, France \\ 3 Equipe Dyliss, Univ Rennes, Inria, CNRS, IRISA, Rennes, France \\ 4 Inria, Bordeaux Sud-Ouest Université, Talence, France
}

Corresponding Authors: Elham Karimi, Simon M. Dittami

Email address: ekarimi@sb-roscoff.fr, simon.dittami@sb-roscoff.fr

Animals, plants, and algae rely on symbiotic microorganisms for their development and functioning. Genome sequencing and genomic analyses of these microorganisms provide opportunities to construct metabolic networks and to analyze the metabolism of the symbiotic communities they constitute. Genome-scale metabolic network reconstructions rest on information gained from genome annotation. As there are multiple annotation pipelines available, the question arises to what extent differences in annotation pipelines impact outcomes of these analyses. Here, we compare five commonly used pipelines (Prokka, MaGe, IMG, DFAST, RAST) from predicted annotation features (coding sequences, Enzyme Commission numbers, hypothetical proteins) to the metabolic network-based analysis of symbiotic communities (biochemical reactions, producible compounds, and selection of minimal complementary bacterial communities). While Prokka and IMG produced the most extensive networks, RAST and DFAST networks produced the fewest false positives and the most connected networks with the fewest dead-end metabolites. Our results underline differences between the outputs of the tested pipelines at all examined levels, with small differences in the draft metabolic networks resulting in the selection of different microbial consortia to expand the metabolic capabilities of the algal host. However, the consortia generated yielded similar predicted producible compounds and could therefore be considered functionally interchangeable. This contrast between selected communities and community functions depending on the annotation pipeline needs to be taken into consideration when interpreting the results of metabolic complementarity analyses. In the future, experimental validation of bioinformatic predictions will likely be crucial to both evaluate and refine the pipelines and needs to be coupled with increased efforts to expand and improve annotations in reference databases.
Peerr reviewing PDF (2020:10:53503:2:0:NEW 2 A Pr 2021) 


\title{
1 Robustness analysis of metabolic predictions in algal microbial communities 2 based on different annotation pipelines
}

3

4

5

6

\author{
Elham Karimi $^{*}$, Enora Geslain ${ }^{1,2}$, Arnaud Belcour ${ }^{3}$, Clémence Frioux ${ }^{4}$, Méziane Aïte ${ }^{3}$, \\ Anne Siegel ${ }^{3}$, Erwan Corre ${ }^{2}$, Simon M. Dittami ${ }^{1}$ * \\ ${ }^{1}$ Sorbonne Université/CNRS, Station Biologique de Roscoff, UMR8227, Integrative Biology of \\ Marine Models, CS 90074, Roscoff, France. \\ ${ }^{2}$ Sorbonne Université/CNRS, Station Biologique de Roscoff, FR2424, CS 90074, Roscoff, \\ France Roscoff, France. \\ ${ }^{3}$ Univ Rennes, Inria, CNRS, IRISA, Equipe Dyliss, Rennes, France. \\ ${ }^{4}$ Inria, Bordeaux Sud-Ouest Université, 33405 Talence, France.
}

Corresponding Authors:

Elham Karimi ${ }^{1} \&$ Simon M. Dittami ${ }^{1}$

Place Georges Teissier, 29680 Roscoff, France

Email address: elham.karimi@sb-roscoff.fr. \& simon.dittami@sb-roscoff.fr.

\begin{abstract}
Animals, plants, and algae rely on symbiotic microorganisms for their development and functioning. Genome sequencing and genomic analyses of these microorganisms provide opportunities to construct metabolic networks and to analyze the metabolism of the symbiotic communities they constitute. Genome-scale metabolic network reconstructions rest on information gained from genome annotation. As there are multiple annotation pipelines available, the question arises to what extent differences in annotation pipelines impact outcomes of these analyses. Here, we compare five commonly used pipelines (Prokka, MaGe, IMG, DFAST, RAST) from predicted annotation features (coding sequences, Enzyme Commission numbers, hypothetical proteins) to the metabolic network-based analysis of symbiotic communities (biochemical reactions, producible compounds, and selection of minimal complementary bacterial communities). While Prokka and IMG produced the most extensive networks, RAST and DFAST networks produced the fewest false positives and the most connected networks with the fewest dead-end metabolites. Our results underline differences between the outputs of the tested pipelines at all examined levels, with small differences in the draft metabolic networks resulting in the selection of different microbial consortia to expand the metabolic capabilities of the algal host. However, the consortia generated yielded similar predicted producible compounds and could therefore be considered functionally interchangeable. This contrast between selected communities and community functions depending on the annotation pipeline needs to be taken into consideration when interpreting the results of metabolic complementarity analyses. In the future, experimental validation of bioinformatic predictions will likely be crucial to both evaluate and refine the pipelines and needs to be coupled with increased efforts to expand and improve annotations in reference databases. Keywords: Gene prediction; functional annotation; genome-scale metabolic networks; metabolic complementary analyses; metabolic exchanges; holobionts
\end{abstract}




\section{Introduction}

46 Plants, animals, and algae are hosts to a large diversity of microorganisms. The importance of

47 these symbiotic microbes for the development and functioning of their hosts is widely accepted

48 (Amin et al. 2015; Fraune \& Bosch 2010; McFall-Ngai et al. 2013; Philippot et al. 2013). This is

49 also true for brown algal surfaces, which provide an attractive substrate for different bacterial

50 phyla, most importantly the Proteobacteria, Bacteroidetes, Firmicutes, and Actinobacteria.

51 These bacteria are important for the brown algal host e.g. by promoting its growth and by

\section{Materials \& Methods} providing defenses against pathogens (KleinJan et al. 2017; Singh \& Reddy 2014). The mutualistic relationships between algae and their microbiota are so tightly interwoven that both parts together are frequently considered a functional entity, the so-called holobiont (Egan et al. 2013).

Recent advances in sequencing technology have generated new opportunities for genome sequencing and genomic analyses of microbial populations (Dittami et al. 2021). A key step in interpreting this wealth of genomic data is their structural and functional annotation, and multiple prokaryotic annotation pipelines have been developed to accomplish this task (Siezen \& van Hijum 2010). Based on functional annotations, genome-scale metabolic networks can then be generated, providing a formal representation of an organisms' metabolism (Haggart et al. 2011). Finally, metabolic complementarity can highlight potentially beneficial metabolic interactions between a host and its symbionts (Dittami et al. 2014; Muller et al. 2018), and the degree of metabolic complementarity between two organisms has been suggested to be a direct indicator of beneficial vs. competitive interaction in a system (Burgunter-Delamare et al. 2020; Levy et al. 2015). It can even be used as a criterion for the selection of minimal consortia where the host attains maximal metabolic potential while minimizing exchanges (Frioux et al. 2018; Levy et al. 2015). Although these tools and pipelines provide a promising framework for a comprehensive understanding of the metabolism of organisms, it is currently unknown to what extent outcomes of such analyses are sensitive to gene-associations present in the metabolic networks they rely on, and thus the precision of the prokaryotic annotation pipelines used. For metabarcoding analyses, for instance, the choice of the bioinformatic pipeline has been shown to have a significant impact on some of the biological conclusions that could be drawn (Siegwald et al. 2019).

Here we compared a selection of widespread genome annotation pipelines, examined their impact on the reconstructed genome-scale metabolic networks, and assessed whether the same conclusion about metabolic complementarity could be reached with the different pipelines. Our work was carried out using the filamentous brown alga Ectocarpus subulatus as a model system. Different strains of Ectocarpus have been established as a genomic and genetic model (Cock et al. 2010) and an increasing amount of information is available on its microbiome (BurgunterDelamare et al. 2020; Dittami et al. 2015; Tapia et al. 2016), including a collection of 72 genomes (Karimi et al. 2019) of bacteria isolated from Ectocarpus as a host (Dittami et al. 2020b).

\section{Genome Annotation}


90

91

92

93

94

95

96

97

98

99

100

101

102

103

104

105

106

107

108

109

110

111

112

113

114

115

116

117

118

119

120

121

122

123

124

125

126

127

128

129

130

131

132

133

134

135

Eighty-one publicly available genomes were used for this study (Table S1), all of them corresponding to strains that have been isolated from the brown alga Ectocarpus subulatus. The genomes were structurally and functionally annotated using five popular annotation pipelines: the Magnifying Genomes (MaGe) platform (Vallenet et al. 2019), the Rapid Annotations using Subsystems Technology (RAST) toolkit (Aziz et al. 2008), the Integrated Microbial Genomes (IMG) server (Markowitz et al. 2009), the Prokka tool v1.13 (Seemann 2014) and the DDBJ Fast Annotation and Submission Tool (DFAST) (Tanizawa et al. 2017). Each pipeline uses one or more unique databases to predict and search for genes and gene functions. MaGe searches for functional features using UniProtKB/Swiss-Prot, Interpro, FIGFAM, COG, ENZYME, and Diamond as a search tool (Vallenet et al. 2019); RAST predicts gene functions using the SEED database (Aziz et al. 2008); IMG predicts features for genes based on COGs, Pfams, TIGRfams, as well as the KEGG and MetaCyc; Prokka is a command-line tool using UniProt, Pfam, and TIGRFAMs; DFAST uses ortholog searches with reciprocal BLAST searches, HMM searches against TIGRFAM, and COG assignments for functional annotations (Tanizawa et al. 2017). All of the pipelines were run with default parameters (Table S2). For Prokka, we also performed tests with more stringent e-value settings (1e-15 vs 1e-6(default)); as these results were similar to those obtained with default parameters they are included only in the supplementary tables. supplementary tables.

\section{Draft metabolic network reconstruction}

The draft metabolic network of the host alga Ectocarpus subulatus was already available (Dittami et al. 2020a) and had been reconstructed using Pathway Tools version 23 (Karp et al. 2019) and the AuReMe pipeline (Aite et al. 2018). To be able to use this metabolic network of the host for complementarity analyses, the same version of Pathway Tools was also used to reconstruct the bacterial draft metabolic networks. For each annotated bacterial genome (i.e. 5 pipelines x 81 strains), a draft metabolic network was automatically reconstructed using Mpwt, the PathoLogic multiprocessing wrapper Python package (Belcour et al. 2020) taking into account the protein description, GO terms, and complete EC numbers. The output files created by Pathway Tools were then converted to the SBML format with the Padmet software. During this process the reactions without gene association were removed. Then, the list of reactions as well as network statistics were extracted using the report_network.py script from the Padmet software (Aite et al. 2018). Consequently, for each of the five annotation pipelines, a set of 81 draft metabolic networks was obtained and used for metabolic complementarity analyses. Furthermore, one additional set was produced for each strain by merging the metabolic networks obtained for each annotation pipeline.

\section{Predicting Metabolic Complementarity}

The SBML files were utilized to predict potential metabolic complementarity between the algal host and the bacteria using the MiSCoTo tool (Frioux et al. 2018). Based on a list of compounds present in the culture medium, i.e. Provasoli-enriched seawater as defined by Prigent et al. (2014), MiSCoTo predicts the production of compounds (i.e. the scope) in combined metabolic networks based on a discrete abstraction, which assumes recursively that a compound is producible either if it belongs to the culture medium if it is the product of a reaction in the

Peer] reviewing PDF | (2020:10:53503:2:0:NEW 2 Apr 2021) 
136 combined metabolic networks whose substrates are themselves producible, as stated in the

137 network expansion algorithm (Ebenhöh et al. 2004). It thus does not consider the kinetics, or

138 stoichiometry of the reactions, nor the relative abundance of the organism corresponding to the

139 metabolic networks. MisCoTo furthermore allows for the selection of minimal symbiont

140 communities that enable the production of a given set of compounds. In our analyses of the data

141 obtained with the five different pipelines individually plus one analysis merging all genomes

142 from all pipelines, the set of host metabolites that became producible through symbiotic

143 cooperation was computed with miscoto_scopes. This set was then used as a target for

144 community selection with miscoto_mincom using "--soup" as an option, i.e. ignoring the

145 putative cost of exchanges associated with ensuring the producibility of these novel metabolites

146 by the host. In each experiment, the minimal community size ensuring the producibility of these

147 algal compounds was computed, together with the set of symbionts that appears in at least one of

148 the minimal communities ("--union" option). Both the list of producible metabolites and the

149 selected microbial communities were compared for all tested annotation pipelines. The detailed

150 description of the code and data used for the above steps is available on Github

151 https://github.com/ElhamKarimi/Metabolic-predictions different-Pipelines.

152

153

154

155

156

157

158

159

160

Indirect measures of network quality and manual examination of pipeline-specific reactions reconstructed metabolic networks

To gain insight into the quality of metabolic networks generated based on the different pipelines, dead-end metabolites were computed for each pipeline using Memote (Lieven et al. 2020). Furthermore, sizes of the largest strongly connected components were counted in each of the networks to obtain a measure of its overall connectivity. First, directed metabolite graphs were constructed from the SBML files using the Networkx package (Hagberg et al. 2008). Then, 23 ubiquitous metabolites were removed (water, proton, ATP, ADP, 3-5-ADP, AMP, NADP, NADPH, NAD, NADH, FAD, UDP, GTP, GDP, oxygen molecule, inorganic phosphate,, diphosphate, carbon dioxide, ammonia, hydrogen peroxide, coenzyme $\mathrm{A}, \mathrm{H}_{2}$-acceptor, $\mathrm{H}_{2}$-donorH2). Finally, for each graph, strongly connected components were found with the Networkx strongly_connected_components function. Then, for each metabolic network, the ratio between the size of the largest strongly connected component and the total number of metabolites was computed.

168 In addition to these indirect measures, we randomly chose 100 reactions that were exclusively

169 found in one pipeline (20 per pipeline) for manual curation. Protein sequences of the bacterial genes that led to the prediction of these reactions were aligned with the Swissprot/Uniprot database using blastP and were then classified as high confidence ( $>30 \%$ ID; $>70 \%$ coverage with a protein with the same metabolic function, functional domains present), low confidence (lower similarity with a protein with the same function, or similarity with a protein with a distinct but similar function), or false ( $>30 \%$ ID $;>70 \%$ coverage with proteins known to carry out a different function).

\section{Statistical Analyses}

All statistical analyses were carried out using RStudio (v.1.1.463). Differences in the number of annotation features per pipeline were determined using an ANOVA followed by a Tukey's HSD (honestly significant difference) test, considering each genome as a replicate. Nonmetric 
183 multidimensional scaling (NMDS) was performed on a presence/absence matrix comprising all 184 reactions for each genome/annotation pipeline. The Bray-Curtis index was used as a measure of 185 quantification of compositional dissimilarity between genomes annotated by different pipelines. 186 To verify if the annotation pipeline had an impact on the reaction, an ANOSIM test (number of 187 permutations $=9999)$ was performed using the Vegan package (Oksanen et al. 2020). UpSet 188 diagrams were generated using the UpSetR package (Conway et al. 2017). Comparisons of the 189 overall numbers of predicted EC-numbers per category were tested using a Binomial test and a

\section{Results}

81 bacterial genomes were annotated using the Prokka, RAST, IMG, MaGe, and DFAST pipelines, and the number of predicted features (Coding Sequences (CDSs), Enzyme Commission (EC) numbers, hypothetical proteins) are shown for each genome and pipeline in Table S1. The number of predicted CDSs across all genomes and pipelines ranged from 2180 to 6755; the number of predicted EC-numbers ranged from 443 to 2106 . The number of hypothetical proteins ranged from 56 to 3566 . Finally, the average number of predicted metabolic reactions, after automatic metabolic network reconstruction with Pathway Tools, ranged from 1034 to 2052. Figure 1 shows the impact of the tested annotation pipelines on these metrics. At the level of CDS prediction, we observed divergence with MaGe, for instance, predicting more CDSs per genome than all other pipelines. Also, MaGe annotation contained fewer unknown hypothetical proteins.

Prokka predicted more EC numbers than the other pipelines, regardless of the e-value cutoff used. The precision of the predictions was similar in Prokka, IMG, MaGe, and DFAST with 85, 88,84 , and $85 \%$ of all EC numbers being complete. The only outlier was RAST, which provided only complete EC numbers (Table S3). We did, however, observe significant differences between the pipelines regarding certain categories of EC numbers. For instance, DFAST predicted most oxidoreductases acting on NADH/NADPH, IMG predicted most Ligases forming phosphoric ester bonds, MAGE was the only pipeline to predict translocases (E.C. 7.-.-.-), PROKKA predicted most hydrolases acting on phosphorus-nitrogen bonds, and RAST predicted most hydrolases acting on ether bonds. A detailed list of overrepresented EC numbers per annotation pipeline is provided in Table 1 .

In terms of predicted metabolic reactions, IMG predicted the highest numbers $(4.2 \%$ more than average) closely followed by Prokka with default settings (3.1\%), while DFAST annotations resulted in the fewest reactions ( $7.7 \%$ fewer than average). Similar patterns were also obtained when running the analyses separately for the different bacterial phyla (Fig. S1), except that ECnumber predictions for Actinobacteria by DFAST were lower compared to other pipelines, and CDS prediction for Firmicutes by IMG were slightly higher. Finally, we examined three indirect measures of quality for the network reconstructions: the percentage of dead-ends, i.e. metabolites which can be produced but are not consumed; orphan metabolites, i.e. metabolites that are consumed but not produced in the metabolic networks; and finally, the ratio between the size of the largest strongly connected components and the total number of metabolites, a direct estimate of the "connectedness" of the network. The percentage of dead-end metabolites was similar across all pipelines: $30.35 \%$ for Prokka, 29.66\% for IMG, 29.17 for DFAST, $29.02 \%$ for MaGe, and $28.4 \%$ for RAST. The percentage of orphan metabolites was also similar: $30.63 \%$ for 
229 Prokka, 30.49\% for IMG, 29.81\% for DFAST, 30.37\% for MaGe, and 29.48\% for RAST. Lastly,

230 this was also true for the ratio between the size of the largest of strongly connected components

231 and the total number of metabolites: 26\% for Prokka, 28\% for IMG, $29 \%$ for DFAST, $27 \%$ for

232 MaGe, and 29\% for RAST.

233 To determine how similar networks based on different annotation pipelines were, the Bray-

234 Curtis dissimilarity, a unidimensional distance measure between two matrices or networks, was

235 calculated for all network comparisons. Non-Metric Multidimensional Scaling (NMDS) was then

236 used to display the resulting distance matrix in the two-dimensional space. In the resulting graph

237 (Fig. 2 and Table S4) the distance between two points represents the dissimilarity of the

238 underlying networks. It shows that the similarity between networks is determined by the bacterial

239

240

241

242

243

244

245

246

247

248

249

250

251

252

253

254

255

256

257

258

259

260

261

262

263

264

265

266

267

268

269

270

271

272

273

274 phyla, and less so by the annotation pipeline, but within each phylum, there was neither a clear clustering according to strain nor according to annotation pipeline. ANOSIM analyses were then used to determine if these patterns were statistically significant. They show that both phylum and annotation pipeline had a significant effect on the similarity of the networks $(\mathrm{p}<0.0001$ in both cases $)$, but confirm that the effect was stronger for phylum $(\mathrm{R}=0.93)$ than for annotation pipeline $(\mathrm{R}=0.41)$.

To further explore the differences in the metabolic network reconstructions and to highlight the specificities of each annotation pipeline we aimed to determine the conserved and unique content between the genome annotations from these different pipelines. This was done using the UpSetR package. Besides generating lists of reactions specific to different subsets of pipelines, this package also generates an UpSet diagram (Fig. 3), which displays the size of these sets. For instance, 2535 reactions were predicted in at least one genome with each of the 5 pipelines. Prokka and MaGe annotation resulted in the most pipeline-specific reactions: 390 (325 with more stringent e-value) and 256 respectively. These two tools also shared a high number of pairwise exclusive metabolic reactions, i.e. reactions not found by any other pipeline (307 vs 1565 for all other pairwise comparisons). The lists of reactions exclusively found in each pipeline as well as lists of the metabolic pathways they belong to are provided in Tables S5 and S6.

To get an overview of the dominant metabolic functions specific to the networks based on the different pipelines, we examined pipeline-specific metabolic reactions at the level of the pathways they participate in. In total, 180 pathways comprised the reactions specific to Prokka, and among them, 28 were constituted of $>50 \%$ Prokka-specific reactions, including nitrobenzene degradation (PWY-5637) I, taurine degradation I (PWY-1263), albaflavenone biosynthesis (PWY-5887), and novobiocin biosynthesis (PWY-7287). Similarly, for MaGe the specific reactions could be associated with 127 pathways, and 11 were constituted of $>50 \%$ specific reactions, including nitroethane degradation (PWY-5355), sulfite oxidation I (PWY-527), and sulfite oxidation IV (PWY-5326). For RAST 99 pathways including 6 pathways $>50 \%$ complete presented the specific reactions, e.g. acetaldehyde biosynthesis II (PWY-6330) and trypanothione biosynthesis (TRYPANOSYN-PWY). IMG-specific reactions represented 52 pathways with only $4>50 \%$ complete, including tRNA splicing II (PWY-7803) and aldoxime degradation (P345-PWY). Finally, reactions predicted specifically by DFAST corresponded to 42 pathways with only Nitrogen fixation II (PWY-7576) and Fatty acid biosynthesis (PWY5970) being $>50 \%$ complete (Table S6).

To evaluate the accuracy of the annotation pipelines, manual curation of 20 randomly selected reactions that were unique to each pipeline was carried out. DFAST has the highest 
275 number of high confidence assignations (10) followed by Prokka and MAGE (6), IMG(4), and

276 RAST(1). RAST, on the other hand, did not produce any annotations that could be clearly

277 identified as false, followed by Prokka (1), DFAST (2), IMG (3), and MaGe (3). In Prokka,

278 increasing the stringency of the e-value cutoff did not affect the proportions of high confidence,

279 low confidence or false reactions. In DFAST, although the correct reaction was associated with

280 the gene, we observed discrepancies between the product name of genes and the EC number

281 associated in 3 cases. In MAGE and IMG we found 1 and 3 predicted proteins without any

282 similarity in the database and classified them as low confidence (Table S7).

283

284

285

286

287

288

289

290

291

292

293

294

295

296

297

298

299

300

301

302

303

304

305

306

307

308

309

310

311

312

313

314

315

316

317

318

319

To assess how these differences in the draft metabolic networks impacted the function of the predicted metabolism of algal-bacterial holobionts, we next examined the list of metabolites that could be produced by the algal metabolic network when combined with the $81 \mathrm{draft}$ bacterial networks for each annotation pipeline (i.e. the predicted producible compounds). As shown in Table 2, based on Prokka, RAST, IMG, MaGe, and DFAST 506, 492, 484, 549, and 504 producible compounds were identified, respectively (see Table S8 for a list of compounds). 448 (i.e. between $81.6 \%$ and $92.6 \%$ of the producible compounds) were shared among all tools, but MaGe, RAST, and Prokka also had 34, 11, and 10 exclusive compounds (Fig. 3B).

Furthermore, 12 additional compounds became producible by merging the networks of all strains and annotation pipelines.

Next, we computed how many strains were needed to enable the production of the same metabolic compounds as the entire bacterial communities for each pipeline (Table 2). As expected, the annotation pipeline affected the number of strains involved in a minimal community, which increased with the number of metabolites that could be produced by the entire bacterial community. The number of selected strains ranged from seven strains needed to produce the 484 metabolites producible by the metabolic networks reconstructed with the IMG pipeline to 13 strains needed to produce the 549 metabolites producible by the MaGe metabolic networks. Fourteen strains were required to produce the full 590 compounds producible by the combination of all networks from all pipelines. We also examined which bacteria were involved in these minimal communities, taking into consideration the union of all minimal communities proposed by MisCoTo. Here, the annotation pipeline had a strong effect on these communities, with strains being selected from sub-sets of bacteria ranging from 15 (Prokka) to 61 (RAST) strains (Table 2). The number of bacteria in each of the sub-sets was not related to the number of producible compounds nor the size of the minimal communities. Fifteen strains were selected in only one of the five pipelines, 14 of which exclusively in the RAST-based community, and one, Pseudomonas 9AG, was included in minimal communities only when they were calculated from merged data from all annotation pipelines. Most strains were jointly selected by two or more pipelines, but even Prokka and IMG, for which minimal communities were based on the smallest subset of bacteria (15 and 17 selected strains, respectively) had only six strains in common. However, we also found commonalities between the selected communities: all communities comprised at least one representative of the Actinomycetales (Actinobacteria), Bacillales (Firmicutes), Rhizobiales (Alphaproteobacteria), Sphingomonadales (Alphaproteobacteria), and Pseudomonadales (Gammaproteobacteria). Lastly, one strain, Maribacter strain 151 (Flavobacteriaceae, Bacteroidetes), was consistently selected in all data sets regardless of the annotation tool (Table 2).

320 
321 Finally, given the high variability between the selected communities, we tested how specific

322 these communities were to the dataset used to select them. To this means, we selected the strains

323 comprised in the minimal microbial communities predicted based on the Prokka annotations,

324 i.e. 15 bacteria, and examined the metabolites predicted to be produced by this community

325 together with the host when applied to the datasets based on the other pipelines. Briefly, the

326 DFAST, IMG, MaGe, and RAST metabolic networks with the Prokka community predicted

327 similar scopes as the larger communities specifically selected for the dataset: 477 compounds vs

328492 for RAST, 469 vs 484 for IMG, 488 vs 549 for MaGe, and 448 vs 504 producible

329 compounds for DFAST, respectively (Table S9). This suggests that differences in the community

330 size and composition between the pipelines can be explained mainly by the need to complement

331

332

333

334

335

336

337

338

339

340

341

342

343

344

345

346

347

348 Our analyses also highlight a number of reactions that were predicted specifically for some

349

350

351

352

353

354

355

356

357

358

359

360

361

362

363

364

365

366

\section{Discussion \& Conclusions}

In this study, we sought to assess the impact of selected annotation pipelines on draft metabolic network reconstructions and downstream analyses of metabolic complementarity. Our results highlight significant differences between the output of the five tested standard pipelines at all examined levels, from the prediction of coding sequences to the selection of microbial communities, especially for EC annotations and hypothetical proteins, with levels of variability similar to those previously reported by Griesemer et al. (2018). Overall, the number of reactions predicted in the final network mirrored the number of EC numbers predicted, with Prokka and IMG yielding both the highest number of EC annotations and reactions. This underlines the importance of this type of annotation for metabolic reconstructions with Pathway Tools - a link that is not surprising as EC numbers are directly referenced in MetaCyc (Caspi et al. 2017), the database used by Pathway Tools for draft metabolic network reconstruction. Each complete EC number can therefore be translated directly into one or several corresponding metabolic reactions. pipelines, but our analysis of the corresponding pathways did not clearly show any pipelines to favor the annotation of specific biological processes over others. Furthermore, the fact that we observed little differences in the relative performance of the pipelines when comparing them separately for different bacterial phyla, suggests that there is no strong phylum-specificity of the tested pipelines. Our manual examination of a subset of pipeline-specific reactions showed that the reliability of these pipeline-specific predictions is generally low, with a tendency for those pipelines that predict the fewest pipeline-specific reactions (DFAST, RAST) to produce the highest proportion of high-confidence prediction, as well as the most connected networks in terms of dead-end metabolites. The overall high proportion of dead-end metabolites can be explained by the facts that i) during the reconstruction process we discarded reactions without genetic support (gene association), and that ii) metabolic networks underwent no or very little curation. Furthermore, the genomes analyzed here belong to non-model organisms, and hence their metabolism is more difficult to reconstruct than in well-established models.

Given these differences, a key question is how reliable functional analyses based on these draft metabolic networks are, and how much their results change according to the annotation pipeline employed. Here our results may seem contradictory at first: on one hand, variability at the level of producible metabolites was low; on the other hand, during the selection of microbial 
367 communities based on metabolic complementarities, even these small differences in the draft

368 metabolic networks resulted in largely different consortia. These small differences, whether they

369 are erroneous or missing annotations, accumulate across the 81 annotated genomes and are

370

371

372

373

374

375

376

377

378

379

380

381

382

383

384

385

386

387

388

389

390

391

392

393

394

395

396

397

398

399

400

401

402

403

404

405

406

407

408

409

410

411 particularly likely to impact the overall set of producible metabolites and hence community selection, as any possible cooperation with an added value to the host is selected. Indeed, although small, differences in the producible metabolites require complementing the bacterial community with strains that specifically enable the production of these metabolites. Overall, however, the low number of pipeline-specific metabolites indicated that all of the selected consortia were functionally similar. Furthermore, we have shown that a given minimal bacterial community (in our case one generated with Prokka data), also yields similar producible metabolites regardless of the annotation pipeline used for the generation of the metabolic networks. This means that the different selected bacterial consortia are likely able to fulfill the same or similar metabolic roles in our metabolic model. This phenomenon has previously also been described in natural alga-associated bacterial consortia (Burke et al. 2011) and was explained by the competitive lottery theory (Sale 1979): several, but not all species/strains can occupy a given niche, and among them, random processes govern which species prevails (lottery model). In our case, such "random" processes could be generated by the 'noise' in the genome annotations and the specificities of each pipeline. Consequently, the niche would correspond to the provision of specific metabolic functions. In line with this analogy, the composition of the bacterial communities in our analyses was not entirely random: although only one strain was present in all communities, each community also contained at least one member of several major taxonomic groups. These groups likely possess specific metabolic capacities absent from other groups making their presence indispensable in all selected consortia. These requirements were detected and met by MiSCoTo regardless of the annotation pipeline.

Regarding the use of metabolic complementarity as a criterion to select microbial communities, this implies that, as also confirmed experimentally by Burgunter-Delamare et al. (2020), metabolic complementarity can be used to select microbial functions important for a symbiotic community. However, we need to expect high variability in the composition of the selected communities if several strains contain similar metabolic capacities. Just like in nature, there may simply not be one ideal solution that clearly excludes all others. In this sense our data does not provide any evidence that one or another pipeline is more suitable for metabolic network reconstruction - we can only state that for our dataset Prokka and IMG, on average, produced more EC numbers and larger draft metabolic networks with Pathway Tools, while DFAST and RAST are likely to produce fewer false-positive reactions and dead-end metabolites, on average. Furthermore, MaGe produced the largest global scope for the community. One approach to avoid biases introduced by annotation pipelines is to merge results from different pipelines thus maximizing the number of annotations (Kalkatawi et al. 2015). Our data obtained for the merged metabolic networks of all pipelines suggest that this approach would also further extend our metabolic networks and the global scope of the community. However, this approach also comes with a risk, as every additional annotation pipeline may introduce additional errors (Poptsova \& Gogarten 2010), especially if the pipeline is not regularly updated (Salzberg 2019). Based on our manual curation of pipelinespecific reactions we may even consider the opposite i.e. basing metabolic complementarity analysis exclusively on reactions that have been predicted independently by two or more pipelines. This will reduce the scope of the networks but likely also result in a reduction of noise and false-

412 positive associations. An additional option may be to increase the stringency of the pipeline 
413 settings. Here moderate modification of the e-value in Prokka had little effect on the final results, 414 but further adjustments are possible and their efficiency would likely need to be adapted for each 415 dataset.

416

417

Another application of metabolic complementarity using different annotation pipeline could also be in the context of metagenome analysis, on Metagenome Assembled Genomes (MAGs) of uncultured bacteria. Belcour et al. (2020) have shown that reconstructed metabolic networks from MAGs are similar to the reference genomes, although the average number of reactions was higher in MAGs. Also, the stability of the producible metabolites and selected minimal symbionts was demonstrated for degraded genomes with $2 \%$ or more of genes randomly removed. It, therefore, seems probable that the overall stability observed in our study in terms of the predicted metabolic contributions of the symbionts to the algal metabolism regardless of the annotation pipeline will also hold true for metagenomic communities.

In the long run, continued and extensive experimental validation of bioinformatic predictions in both a culture and a metagenomic context will be key to evaluate and refine the pipelines (Poptsova \& Gogarten 2010) and needs to be coupled with increased efforts to expand and improve annotations in reference databases (Carr \& Borenstein 2014). In the meantime, one approach to overcome the variability brought by the annotation pipelines is to carefully curate the resulting metabolic networks. This is an indispensable step in obtaining high-quality metabolic networks as stated by Thiele \& Palsson (2010). However, this step is costly as it usually requires human expertise and thorough literature exploration. As more and more genomes and metagenomes are available, there is a need for curation-free and reliable metabolic networks to surmount this bottleneck. Non-curated metabolic networks can be informative but are likely to contain specific possibly false-positive functions brought by the annotation, these functions deserve to be examined closely when used for selecting communities of interest.

\section{Acknowledgments}

We would like to thank Alireza Asvadi for his help writing a python script for merging tables of all strains across pipelines, and the reviewers of this paper for their constructive remarks. We are grateful to the Institut Français de Bioinformatique (ANR-11-INBS-0013) and BioGenouest Roscoff Bioinformatics platform ABiMS (http://abims.sb-roscoff.fr) for providing computing resources.

\section{References}

Aite M, Chevallier M, Frioux C, Trottier C, Got J, Cortés MP, Mendoza SN, Carrier G, Dameron O, Guillaudeux N, Latorre M, Loira N, Markov GV, Maass A, and Siegel A. 2018. Traceability, reproducibility and wiki-exploration for "à-la-carte" reconstructions of genome-scale metabolic models. PLoS Computational Biology 14:e1006146. 10.1371/journal.pcbi.1006146 
456

457

458

459

460

461

462

463

464

465

466

467

468

469

470

471

472

473

474

475

476

477

478

479

480

481

482

483

484

485

486

487

488

489

490

491

492

493

494

495

496

497

498

499

500

501
Amin SA, Hmelo LR, van Tol HM, Durham BP, Carlson LT, Heal KR, Morales RL, Berthiaume CT, Parker MS, Djunaedi B, Ingalls AE, Parsek MR, Moran MA, and Armbrust EV. 2015. Interaction and signalling between a cosmopolitan phytoplankton and associated bacteria. Nature 522:98-101. 10.1038/nature14488

Aziz RK, Bartels D, Best AA, DeJongh M, Disz T, Edwards RA, Formsma K, Gerdes S, Glass EM, and Kubal M. 2008. The RAST server: rapid annotations using subsystems technology. BMC Genomics 9:1. 10.1186/1471-2164-9-75

Belcour A, Frioux C, Aite M, Bretaudeau A, Hildebrand F, and Siegel A. 2020. Metage2Metabo, microbiota-scale metabolic complementarity for the identification of key species. eLife 9:e61968. 10.7554/eLife.61968.

Burgunter-Delamare B, KleinJan H, Frioux C, Fremy E, Wagner M, Corre E, Le Salver A, Leroux C, Leblanc C, Boyen C, Siegel A, and Dittami SM. 2020. Metabolic Complementarity Between a Brown Alga and Associated Cultivable Bacteria Provide Indications of Beneficial Interactions. Frontiers in Marine Science 7:85. 10.3389/fmars.2020.00085.

Burke C, Steinberg P, Rusch D, Kjelleberg S, and Thomas T. 2011. Bacterial community assembly based on functional genes rather than species. Proceedings of the National Academy of Sciences 108:14288-14293. 10.1073/pnas.1101591108.

Carr R, and Borenstein E. 2014. Comparative Analysis of Functional Metagenomic Annotation and the Mappability of Short Reads. PLoS ONE 9:e105776. 10.1371/journal.pone.0105776.

Caspi R, Billington R, Fulcher CA, Keseler IM, Kothari A, Krummenacker M, Latendresse M, Midford PE, Ong Q, Ong WK, Paley S, Subhraveti P, and Karp PD. 2017. The MetaCyc database of metabolic pathways and enzymes. Nucleic Acids Research 46:D633-D639. 10.1093/nar/gkx935.

Cock JM, Sterck L, Rouzé P, Scornet D, Allen AE, Amoutzias G, Anthouard V, Artiguenave F, Aury J-M, Badger JH, Beszteri B, Billiau K, Bonnet E, Bothwell JH, Bowler C, Boyen C, Brownlee C, Carrano CJ, Charrier B, Cho GY, Coelho SM, Collén J, Corre E, Da Silva C, Delage L, Delaroque N, Dittami SM, Doulbeau S, Elias M, Farnham G, Gachon CMM, Gschloessl B, Heesch S, Jabbari K, Jubin C, Kawai H, Kimura K, Kloareg B, Küpper FC, Lang D, Le Bail A, Leblanc C, Lerouge P, Lohr M, Lopez PJ, Martens C, Maumus F, Michel G, Miranda-Saavedra D, Morales J, Moreau H, Motomura T, Nagasato C, Napoli CA, Nelson DR, Nyvall-Collén P, Peters AF, Pommier C, Potin P, Poulain J, Quesneville H, Read B, Rensing SA, Ritter A, Rousvoal S, Samanta M, Samson G, Schroeder DC, Ségurens B, Strittmatter M, Tonon T, Tregear JW, Valentin K, von Dassow P, Yamagishi T, Van de Peer Y, and Wincker P. 2010. The Ectocarpus genome and the independent evolution of multicellularity in brown algae. Nature 465:617. 10.1038/nature09016.

Conway JR, Lex A, and Gehlenborg N. 2017. UpSetR: an R package for the visualization of intersecting sets and their properties. Bioinformatics 33:2938-2940. 10.1093/bioinformatics/btx364.

Dittami SM, Arboleda E, Auguet J-C, Bigalke A, Briand E, Cárdenas P, Cardini U, Decelle J, Engelen A, Eveillard D, Gachon CMM, Griffiths S, Harder T, Kayal E, Kazamia E, Lallier FH, Media M, Marzinelli EM, Morganti T, Núñez Pons L, Prado S, Pintado J, Saha M, Selosse M-A, Skillings D, Stock W, Sunagawa S, Toulza E, Vorobev A, Leblanc C, and Not F. 2021. A community perspective on the concept of marine

Peer) reviewing PDF | (2020:10:53503:2:0:NEW 2 Apr 2021) 
502

503

504

505

506

507

508

509

510

511

512

513

514

515

516

517

518

519

520

521

522

523

524

525

526

527

528

529

530

531

532

533

534

535

536

537

538

539

540

541

542

543

544

545

546

547

holobionts: state-of-the-art, challenges, and future directions. PeerJ

9:e10911.10.7717/peerj.1091.

Dittami SM, Corre E, Brillet-Guéguen L, Lipinska AP, Pontoizeau N, Aite M, Avia K, Caron C, Cho CH, Collén J, Cormier A, Delage L, Doubleau S, Frioux C, Gobet A, GonzálezNavarrete I, Groisillier A, Hervé C, Jollivet D, KleinJan H, Leblanc C, Liu X, Marie D, Markov GV, Minoche AE, Monsoor M, Pericard P, Perrineau M-M, Peters AF, Siegel A, Siméon A, Trottier C, Yoon HS, Himmelbauer H, Boyen C, and Tonon T. 2020a. The genome of Ectocarpus subulatus - A highly stress-tolerant brown alga. Marine Genomics 52:100740. 10.1016/j.margen.2020.100740.

Dittami SM, Duboscq-Bidot L, Perennou M, Gobet A, Corre E, Boyen C, and Tonon T. 2015. Host-microbe interactions as a driver of acclimation to salinity gradients in brown algal cultures. The ISME journal 10:51. 10.1038/ismej.2015.104.

Dittami SM, Eveillard D, and Tonon T. 2014. A metabolic approach to study algal-bacterial interactions in changing environments. Molecular Ecology 23:1656-1660. $10.1111 / \mathrm{mec} .12670$.

Dittami SM, Peters AF, West JA, Cariou T, KleinJan H, Burgunter-Delamare B, Prechoux A, Egan S, and Boyen C. 2020b. Revisiting Australian Ectocarpus subulatus (Phaeophyceae) From the Hopkins River: Distribution, Abiotic Environment, and Associated Microbiota. Journal of Phycology 56:719-729. 10.1111/jpy.12970.

Ebenhöh O, Handorf T, and Heinrich R. 2004. Structural analysis of expanding metabolic networks. Genome Inform 15:35-45.

Egan S, Harder T, Burke C, Steinberg P, Kjelleberg S, and Thomas T. 2013. The seaweed holobiont: understanding seaweed-bacteria interactions. FEMS Microbiology Reviews 37:462-476. 10.1111/1574-6976.12011.

Fraune S, and Bosch TCG. 2010. Why bacteria matter in animal development and evolution. Bioessays 32:571-580. 10.1002/bies.200900192.

Frioux C, Fremy E, Trottier C, and Siegel A. 2018. Scalable and exhaustive screening of metabolic functions carried out by microbial consortia. Bioinformatics 34:i934-i943. 10.1093/bioinformatics/bty588.

Griesemer M, Kimbrel JA, Zhou CE, Navid A, and D'haeseleer P. 2018. Combining multiple functional annotation tools increases coverage of metabolic annotation. BMC Genomics 19:948. 10.1186/s12864-018-5221-9.

Hagberg A, Swart P, and S Chult D. 2008. Exploring network structure, dynamics, and function using networkx. Proceedings of the 7th Python in Science Conference (SciPy2008),:1118.

Haggart CR, Bartell JA, Saucerman JJ, and Papin JA. 2011. Chapter twenty-one - WholeGenome Metabolic Network Reconstruction and Constraint-Based Modeling. In: Jameson D, Verma M, and Westerhoff HV, eds. Methods in Enzymology: Academic Press, 411-433.

Kalkatawi M, Alam I, and Bajic VB. 2015. BEACON: automated tool for Bacterial GEnome Annotation ComparisON. BMC Genomics 16:616. 10.1186/s12864-015-1826-4.

Karimi E, Geslain E, KleinJan H, Tanguy G, Legeay E, Corre E, and Dittami SM. 2019. Genome Sequences of 72 Bacterial Strains Isolated from Ectocarpus subulatus: A Resource for Algal Microbiology. Genome Biology and Evolution 12:3647-3655. 10.1093/gbe/evz278.

Karp PD, Midford PE, Billington R, Kothari A, Krummenacker M, Latendresse M, Ong WK, Subhraveti P, Caspi R, Fulcher C, Keseler IM, and Paley SM. 2019. Pathway Tools 
548

549

550

551

552

553

554

555

556

557

558

559

560

561

562

563

564

565

566

567

568

569

570

571

572

573

574

575

576

577

578

579

580

581

582

583

584

585

586

587

588

589

590

591

592

version 23.0 update: software for pathway/genome informatics and systems biology. Briefings in Bioinformatics 8:bbz104. 10.1093/bib/bbz104.

KleinJan H, Jeanthon C, Boyen C, and Dittami SM. 2017. Exploring the cultivable Ectocarpus microbiome. Frontiers in Microbiology 8:2456. 10.3389/fmicb.2017.02456.

Levy R, Carr R, Kreimer A, Freilich S, and Borenstein E. 2015. NetCooperate: a network-based tool for inferring host-microbe and microbe-microbe cooperation. BMC Bioinformatics 16:164-164. 10.1186/s12859-015-0588-y.

Lieven C, Beber ME, Olivier BG, Bergmann FT, Ataman M, Babaei P, Bartell JA, Blank LM, Chauhan S, Correia K, Diener C, Dräger A, Ebert BE, Edirisinghe JN, Faria JP, Feist AM, Fengos G, Fleming RMT, García-Jiménez B, Hatzimanikatis V, van Helvoirt W, Henry CS, Hermjakob H, Herrgård MJ, Kaafarani A, Kim HU, King Z, Klamt S, Klipp E, Koehorst JJ, König M, Lakshmanan M, Lee D-Y, Lee SY, Lee S, Lewis NE, Liu F, Ma H, Machado D, Mahadevan R, Maia P, Mardinoglu A, Medlock GL, Monk JM, Nielsen J, Nielsen LK, Nogales J, Nookaew I, Palsson BO, Papin JA, Patil KR, Poolman M, Price ND, Resendis-Antonio O, Richelle A, Rocha I, Sánchez BJ, Schaap PJ, Malik Sheriff RS, Shoaie S, Sonnenschein N, Teusink B, Vilaça P, Vik JO, Wodke JAH, Xavier JC, Yuan Q, Zakhartsev M, and Zhang C. 2020. MEMOTE for standardized genomescale metabolic model testing. Nature Biotechnology 38:272-276. 10.1038/s41587-0200446-y.

Markowitz VM, Mavromatis K, Ivanova NN, Chen IA, Chu K, and Kyrpides NC. 2009. IMG ER: a system for microbial genome annotation expert review and curation. Bioinformatics 25:2271-2278. 10.1093/bioinformatics/btp393.

McFall-Ngai M, Hadfield MG, Bosch TCG, Carey HV, Domazet-Lošo T, Douglas AE, Dubilier N, Eberl G, Fukami T, Gilbert SF, Hentschel U, King N, Kjelleberg S, Knoll AH, Kremer N, Mazmanian SK, Metcalf JL, Nealson K, Pierce NE, Rawls JF, Reid A, Ruby EG, Rumpho M, Sanders JG, Tautz D, and Wernegreen JJ. 2013. Animals in a bacterial world, a new imperative for the life sciences. Proceedings of the National Academy of Sciences 110:3229-3236. 10.1073/pnas.1218525110.

Muller EEL, Faust K, Widder S, Herold M, Martínez Arbas S, and Wilmes P. 2018. Using metabolic networks to resolve ecological properties of microbiomes. Current Opinion in Systems Biology 8:73-80. 10.1016/j.coisb.2017.12.004.

Oksanen J, Blanchet F, Friendly M, Kindt R, Legendre P, McGlinn D, Minchin P, O’Hara R, Simpson G, and Solymos P. 2020. Vegan: Community Ecology Package. R package version 2.5-5. 2019. https://CRAN.R-project.org/package=vegan.

Philippot L, Raaijmakers JM, Lemanceau P, and van der Putten WH. 2013. Going back to the roots: the microbial ecology of the rhizosphere. Nature Reviews Microbiology 11:789799. 10.1038/nrmicro3109.

Poptsova MS, and Gogarten JP. 2010. Using comparative genome analysis to identify problems in annotated microbial genomes. Microbiology 156:1909-1917. 10.1099/mic.0.033811-0.

Prigent S, Collet G, Dittami SM, Delage L, Ethis de Corny F, Dameron O, Eveillard D, Thiele S, Cambefort J, Boyen C, Siegel A, and Tonon T. 2014. The genome-scale metabolic network of Ectocarpus siliculosus (EctoGEM): a resource to study brown algal physiology and beyond. The Plant Journal 80:367-381. 10.1111/tpj.12627.

Sale PF. 1979. Recruitment, loss and coexistence in a guild of territorial coral reef fishes. Oecologia 42:159-177. 10.1007/BF00344855. 
593 Salzberg SL. 2019. Next-generation genome annotation: we still struggle to get it right. Genome Biology 20:92. 10.1186/s13059-019-1715-2. 10.1093/bioinformatics/btu153.

Siegwald L, Caboche S, Even G, Viscogliosi E, Audebert C, and Chabé M. 2019. The Impact of Bioinformatics Pipelines on Microbiota Studies: Does the Analytical "Microscope" Affect the Biological Interpretation? Microorganisms 7:393.

Siezen RJ, and van Hijum SAFT. 2010. Genome (re-)annotation and open-source annotation pipelines. Microbial biotechnology 3:362-369. 10.1111/j.1751-7915.2010.00191.x.

Singh RP, and Reddy CRK. 2014. Seaweed-microbial interactions: key functions of seaweedassociated bacteria. FEMS Microbiology Ecology 88:213-230. 10.1111/15746941.12297.

Tanizawa Y, Fujisawa T, and Nakamura Y. 2017. DFAST: a flexible prokaryotic genome annotation pipeline for faster genome publication. Bioinformatics 34:1037-1039. 10.1093/bioinformatics/btx713.

Tapia JE, González B, Goulitquer S, Potin P, and Correa JA. 2016. Microbiota influences morphology and reproduction of the brown alga Ectocarpus sp. Frontiers in Microbiology 7:197. 10.3389/fmicb.2016.00197.

Thiele I, and Palsson BØ. 2010. A protocol for generating a high-quality genome-scale metabolic reconstruction. Nature Protocols 5:93-121. 10.1038/nprot.2009.203.

Vallenet D, Calteau A, Dubois M, Amours P, Bazin A, Beuvin M, Burlot L, Bussell X, Fouteau S, Gautreau G, Lajus A, Langlois J, Planel R, Roche D, Rollin J, Rouy Z, Sabatet V, and Médigue C. 2019. MicroScope: an integrated platform for the annotation and exploration of microbial gene functions through genomic, pangenomic and metabolic comparative

618 analysis. Nucleic Acids Research 48:D579-D589. 10.1093/nar/gkz926. 


\section{Figure 1}

Boxplot of the number of A) CDSs, B) EC-numbers (considers all EC-number), C) Hypothetical proteins, and D) Metabolic reactions of all annotated bacterial species through the five pipelines.

The graphs show the deviation of each genome annotated with every one pipeline from the mean across all pipelines in \%. The thick horizontal line represents the median of the distribution across all genomes for that pipeline, and circles represent outlier data. Letters above the box-plots indicate statistically significant differences (Tukey's HSD test). Pipelines share the same letter, the differences between them are not significant $(p>=0.05)$. (See Table S10 for details)
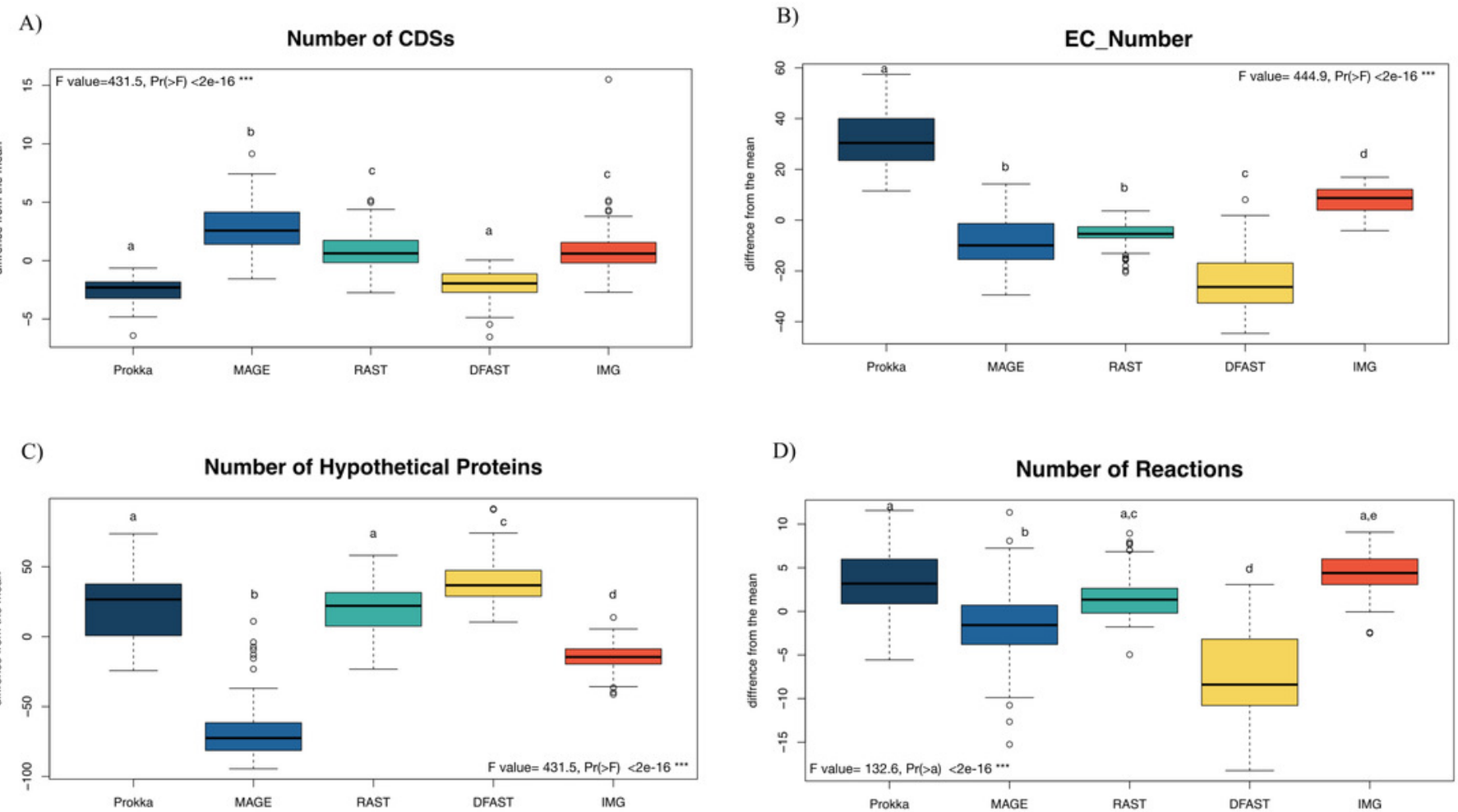
Figure 2

Non-metric Multi-Dimensional Scaling (NMDS) plot of the metabolic networks of the different bacterial strains and pipelines.

The Bray-Curtis index was used as a dissimilarity measure; ellipses show clusters of strains based on bacterial phyla; grey polygons connect annotations of the same genome performed with different pipelines.

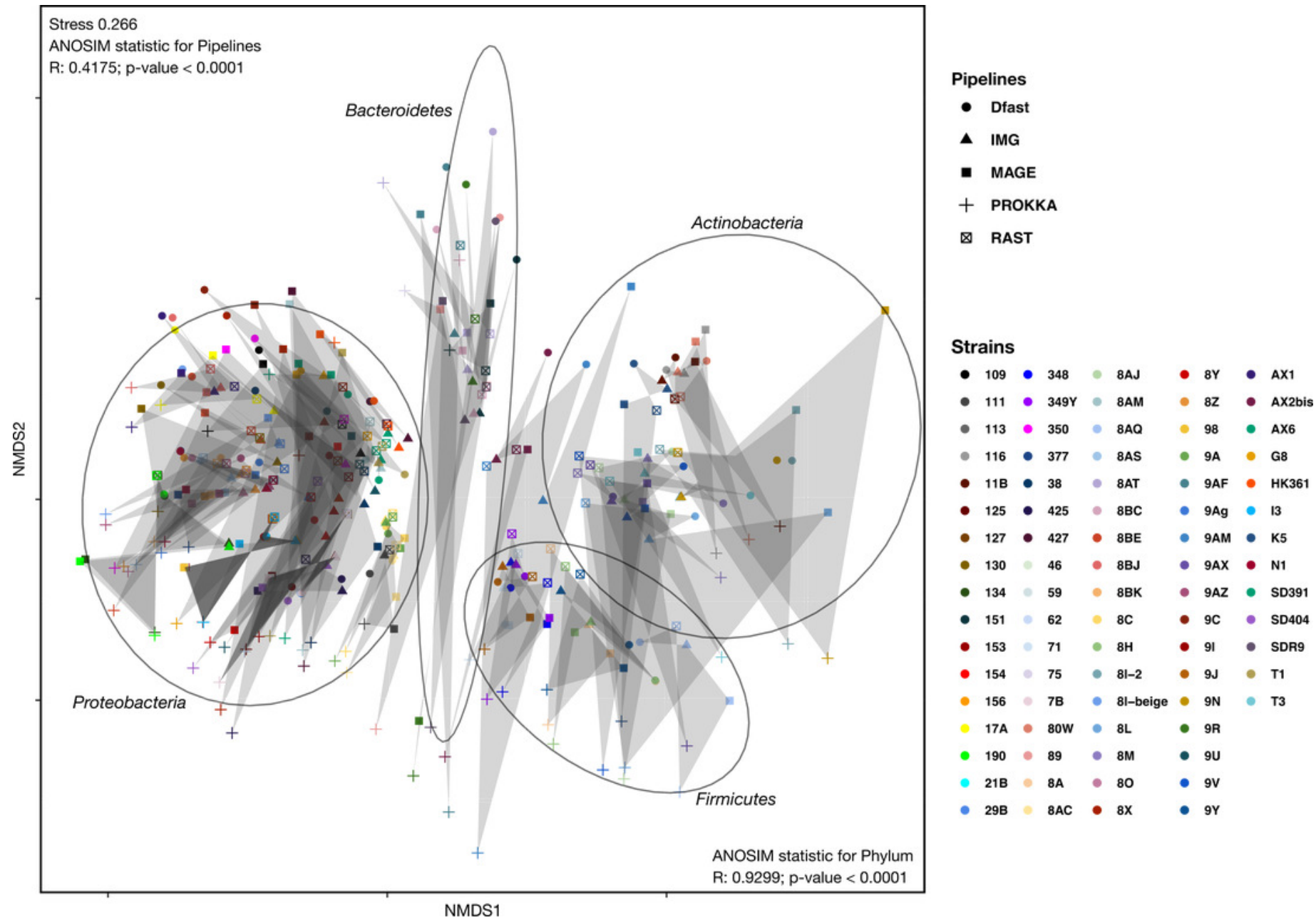




\section{Figure 3}

Upset chart showing the overlap in predicted biochemical reactions $(A)$ and producible compounds by the algal host and the complete set of 81 bacteria (scope) (B) annotated with each pipeline.

The horizontal bars on the left show the total number of predicted reactions (A) or producible compounds (B) in each pipeline. The vertical histogram on the right shows the number of overlapping reactions (A) or compounds (B). The total number of reactions and producible compounds for each tool is indicated on the left as 'set size per tool'. 
A)

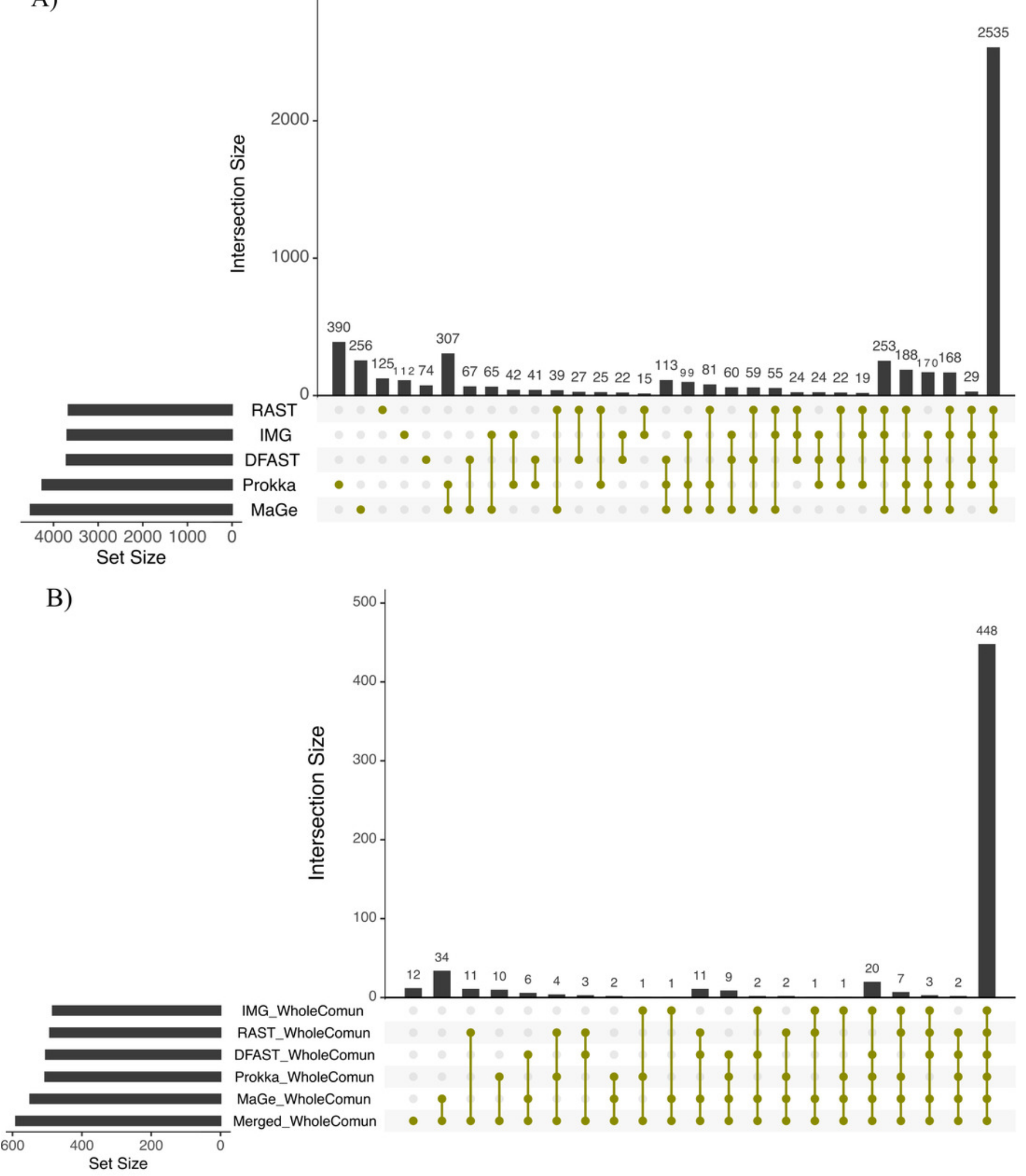




\section{Table $\mathbf{1}$ (on next page)}

Overrepresented EC numbers in the tested pipelines compared to the average of all pipelines.

The "\%over" indicates how many more EC numbers were found in a specific pipeline compared to all pipelines; " $\infty$ " indicates that the EC category was predicted only in this pipeline. p-values correspond to a binomial test after Bonferroni correction. 


\begin{tabular}{|c|c|c|c|}
\hline & Description & \%over & p \\
\hline \multirow[t]{6}{*}{ DFAST } & 1.6 Oxidoreductases acting on NADH or NADPH & $52 \%$ & $<0.001$ \\
\hline & 2.1 Transferase transferring one-carbon groups & $12 \%$ & $<0.001$ \\
\hline & 2.7 Transferase transferring phosphorus-containing groups & $8 \%$ & $<0.001$ \\
\hline & 3.6 Hydrolases acting on acid anhydrides & $22 \%$ & $<0.001$ \\
\hline & 6.1 Ligases forming carbon-oxygen bonds & $17 \%$ & $<0.001$ \\
\hline & 6.3 Ligases forming carbon-nitrogen bonds & $11 \%$ & $<0.001$ \\
\hline \multirow[t]{5}{*}{ IMG } & 2.1 Transferase transferring one-carbon groups & $7 \%$ & 0.019 \\
\hline & 3.1 Hydrolases acting on ester bonds & $9 \%$ & $<0.001$ \\
\hline & 3.4 Hydrolases acting on peptide bonds & $14 \%$ & $<0.001$ \\
\hline & 3.6 Hydrolases acting on acid anhydrides & $15 \%$ & $<0.001$ \\
\hline & 6.5 Ligases forming phosphoric ester bonds & $68 \%$ & $<0.001$ \\
\hline \multirow[t]{8}{*}{ MaGe } & 1.19 Oxidoreductases acting on reduced flavodoxin as donor & $5658 \%$ & $<0.001$ \\
\hline & 4.3 Carbon-nitrogen lyases & $17 \%$ & 0.036 \\
\hline & 5.6 Isomerases altering macromolecular conformation & $\infty$ & $<0.001$ \\
\hline & 7.1 Translocases catalysing the translocation of hydrons & $\infty$ & $<0.001$ \\
\hline & 7.2 Translocases catalysing the translocation of inorganic cations and chelates & $\infty$ & $<0.001$ \\
\hline & 7.3 Translocases catalysing the translocation of inorganic anions & $\infty$ & $<0.001$ \\
\hline & 7.5 Translocases catalysing the translocation of carbohydrates and derivatives & $\infty$ & $<0.001$ \\
\hline & 7.6 Translocases catalysing the translocation of other compounds & $\infty$ & $<0.001$ \\
\hline \multirow[t]{15}{*}{ Prokka } & 1.1 Oxidoreductases acting on $\mathrm{CH}-\mathrm{OH}$ group of donors & $10 \%$ & $<0.001$ \\
\hline & 1.10 Oxidoreductases acting on diphenols and related substances as donors & $56 \%$ & $<0.001$ \\
\hline & 1.12 Oxidoreductases acting on hydrogen as donors & $196 \%$ & $<0.001$ \\
\hline & 1.13 Oxygenases & $37 \%$ & $<0.001$ \\
\hline & 1.14 Oxidoreductases acting on paired donors, with oxygen & $78 \%$ & $<0.001$ \\
\hline & 1.16 Oxidoreductases oxidizing metal ions & $56 \%$ & $<0.001$ \\
\hline & 2.4 Glycosyltransferases & $21 \%$ & $<0.001$ \\
\hline & 2.8 Transferase transferring sulfur-containing groups & $16 \%$ & 0.004 \\
\hline & 3.1 Hydrolases acting on ester bonds & $15 \%$ & $<0.001$ \\
\hline & 3.2 Glycosylases & $15 \%$ & 0.004 \\
\hline & 3.4 Hydrolases acting on peptide bonds & $9 \%$ & 0.025 \\
\hline & 3.6 Hydrolases acting on acid anhydrides & $12 \%$ & $<0.001$ \\
\hline & 3.8 Hydrolases acting on halide bonds & $86 \%$ & $<0.001$ \\
\hline & 3.9 Hydrolases acting on phosphorus-nitrogen bonds & $856 \%$ & $<0.001$ \\
\hline & 4.5 Carbon-halide lyases & $\infty$ & $<0.001$ \\
\hline \multirow[t]{12}{*}{ RAST } & 1.2 Oxidoreductases acting on the aldehyde or oxo group of donors & $18 \%$ & $<0.001$ \\
\hline & 2.3 Acyltransferases & $11 \%$ & $<0.001$ \\
\hline & 2.7 Transferase transferring phosphorus-containing groups & $11 \%$ & $<0.001$ \\
\hline & 3.3 Hydrolases acting on ether bonds & $72 \%$ & $<0.001$ \\
\hline & 4.1 Lyases Carbon-carbon lyases & $15 \%$ & $<0.001$ \\
\hline & 4.2 Carbon-oxygen lyases & $12 \%$ & $<0.001$ \\
\hline & 5.1 Isomerases - racemases, epimerases & $17 \%$ & 0.008 \\
\hline & 5.3 Isomerases - intramolecular oxidoreductases & $16 \%$ & 0.004 \\
\hline & 5.4 Isomerases - intramolecular transferases & $28 \%$ & $<0.001$ \\
\hline & 6.1 Ligases forming carbon-oxygen bonds & $12 \%$ & 0.010 \\
\hline & 6.3 Ligases forming carbon-nitrogen bonds & $10 \%$ & 0.002 \\
\hline & 6.4 Ligases forming carbon-carbon bonds & $41 \%$ & $<0.001$ \\
\hline
\end{tabular}


Table 2 (on next page)

Selection of minimal bacterial communities based on annotation pipelines. 


\begin{tabular}{|c|c|c|c|c|c|c|c|}
\hline Strains & Phylogeny & $\begin{array}{l}\text { Pro- } \\
\text { kka }\end{array}$ & IMG & $\begin{array}{c}\text { Ma- } \\
\text { Ge }\end{array}$ & $\begin{array}{l}\text { DF- } \\
\text { AST }\end{array}$ & $\begin{array}{l}\text { RA- } \\
\text { ST }\end{array}$ & $\begin{array}{c}\text { Mer- } \\
\text { ged }\end{array}$ \\
\hline Curtobacterium sp. 8I-2 & Actinobacteria(1); Actinobacteria(1); Actinomycetales(1); Microbacteriaceae(1); Curtobacterium(1); & & & & & $\checkmark$ & $\checkmark$ \\
\hline Microbacterium sp. 8M & Actinobacteria(1); Actinobacteria(1); Actinomycetales(1); Microbacteriaceae(1); Microbacterium(1); & & & & & $\checkmark$ & $\checkmark$ \\
\hline Plantibacter sp. T3 & Actinobacteria(1); Actinobacteria(1); Actinomycetales(1); Microbacteriaceae(1); Plantibacter(1); & & & & & $\checkmark$ & $\checkmark$ \\
\hline Pseudoclavibacter sp. 8L & Actinobacteria(1); Actinobacteria(1); Actinomycetales(1); Microbacteriaceae(1); Pseudoclavibacter(1); & $\checkmark$ & & & $\checkmark$ & $\checkmark$ & $\checkmark$ \\
\hline Arthrobacter sp. 8AJ & Actinobacteria(1); Actinobacteria(1); Actinomycetales(1); Micrococcaceae(1); Arthrobacter(1); & & & & & $\checkmark$ & $\checkmark$ \\
\hline Arthrobacter sp. 9V & Actinobacteria(1); Actinobacteria(1); Actinomycetales(1); Micrococcaceae(1); Arthrobacter(1); & & $\checkmark$ & & & & $\checkmark$ \\
\hline Citricoccus sp. K5 & Actinobacteria(1); Actinobacteria(1); Actinomycetales(1); Micrococcaceae(1); Citricoccus(1); & & & $\checkmark$ & & $\checkmark$ & $\checkmark$ \\
\hline Micrococcus sp. 116 & Actinobacteria(1); Actinobacteria(1); Actinomycetales(1); Micrococcaceae(1); Micrococcus(1); & $\checkmark$ & & & & $\checkmark$ & $\checkmark$ \\
\hline Micrococcus sp. 11B & Actinobacteria(1); Actinobacteria(1); Actinomycetales(1); Micrococcaceae(1); Micrococcus(1); & & & & & $\checkmark$ & $\checkmark$ \\
\hline Micrococcus sp. 80W & Actinobacteria(1); Actinobacteria(1); Actinomycetales(1); Micrococcaceae(1); Micrococcus(1); & & & & & $\checkmark$ & $\checkmark$ \\
\hline Aeromicrobium sp. 9AM & Actinobacteria(1); Actinobacteria(1); Actinomycetales(1); Nocardioidaceae(1); Aeromicrobium(1); & $\checkmark$ & & & & $\checkmark$ & $\checkmark$ \\
\hline Nocardioides sp. AX2bis & Actinobacteria(1); Actinobacteria(1); Actinomycetales(1); Nocardioidaceae(1); Nocardioides(1); & & $\checkmark$ & & & $\checkmark$ & $\checkmark$ \\
\hline Imperialibacter sp. 89 & Bacteroidetes(1); Cytophagia(1); Cytophagales(1); Flammeovirgaceae(1); Imperialibacter(1); & & & $\checkmark$ & $\sqrt{ }$ & & $\sqrt{ }$ \\
\hline Imperialibacter sp. EC-SDR9 & Bacteroidetes(1); Cytophagia(1); Cytophagales(1); Flammeovirgaceae(1); Imperialibacter(1); & & & $\checkmark$ & $\checkmark$ & & \\
\hline Imperialibacter sp. SDR9 & Bacteroidetes(1); Cytophagia(1); Cytophagales(1); Flammeovirgaceae(1); Imperialibacter(1); & & & $\checkmark$ & $\checkmark$ & & $\checkmark$ \\
\hline Flavobacterium sp. 9R & Bacteroidetes(1); Flavobacteriia(1); Flavobacteriales(1); Flavobacteriaceae(1); Flavobacterium(1); & $\checkmark$ & $\checkmark$ & & & $\checkmark$ & $\checkmark$ \\
\hline Frigoribacterium sp. 9N & Bacteroidetes(1); Flavobacteriia(1); Flavobacteriales(1); Flavobacteriaceae(1); Flavobacterium(1); & $\checkmark$ & & & & $\checkmark$ & $\checkmark$ \\
\hline Maribacter sp. 151 & Bacteroidetes(1); Flavobacteriia(1); Flavobacteriales(1); Flavobacteriaceae(1); Maribacter(1); & $\checkmark$ & $\checkmark$ & $\checkmark$ & $\checkmark$ & $\checkmark$ & $\checkmark$ \\
\hline Sphingobacterium sp. 8BC & Bacteroidetes(1); Sphingobacteriia(1); Sphingobacteriales(1); Sphingobacteriaceae(1); Sphingobacterium(1); & & & & & $\checkmark$ & $\checkmark$ \\
\hline Bacillus sp. 348 & Firmicutes(1); Bacilli(1); Bacillales(1); Bacillaceae 1(1); Bacillus(1); & & & & & $\checkmark$ & \\
\hline Bacillus sp. 349Y & Firmicutes(1); Bacilli(1); Bacillales(1); Bacillaceae 1(1); Bacillus(1); & & & & $\checkmark$ & $\checkmark$ & \\
\hline Bacillus sp. 71 & Firmicutes(1); Bacilli(1); Bacillales(1); Bacillaceae 1(1); Bacillus(1); & & & & & $\checkmark$ & \\
\hline Bacillus sp. 9J & Firmicutes(1); Bacilli(1); Bacillales(1); Bacillaceae 1(1); Bacillus(1); & $\checkmark$ & & & & $\checkmark$ & \\
\hline Exiguobacterium sp. 8A & Firmicutes(1); Bacilli(1); Bacillales(1); Bacillales_Incertae Sedis XII(1); Exiguobacterium(1); & & & & & $\checkmark$ & $\checkmark$ \\
\hline Exiguobacterium sp. 8H & Firmicutes(1); Bacilli(1); Bacillales(1); Bacillales_Incertae Sedis XII(1); Exiguobacterium(1); & & & & & $\checkmark$ & $\checkmark$ \\
\hline Exiguobacterium sp. 9Y & Firmicutes(1); Bacilli(1); Bacillales(1); Bacillales_Incertae Sedis XII(1); Exiguobacterium(1); & & & & & $\checkmark$ & $\checkmark$ \\
\hline Flavobacterium sp. $9 \mathrm{AF}$ & Firmicutes(1); Bacilli(1); Bacillales(1); Bacillales_Incertae Sedis XII(1); Exiguobacterium(1); & $\checkmark$ & $\checkmark$ & $\checkmark$ & & $\checkmark$ & $\checkmark$ \\
\hline Staphylococcus sp. 8AQ & Firmicutes(1); Bacilli(1); Bacillales(1); Staphylococcaceae(1); Staphylococcus(1); & & $\checkmark$ & $\checkmark$ & & $\checkmark$ & $\checkmark$ \\
\hline Brevundimonas sp. G8 & Proteobacteria(1); Alphaproteobacteria(1); Caulobacterales(1); Caulobacteraceae(1); Brevundimonas(1); & & & & & $\checkmark$ & \\
\hline Bosea sp. 125 & Proteobacteria(1); Alphaproteobacteria(1); Rhizobiales(1); Bradyrhizobiaceae(1); Bosea(1); & & & & $\checkmark$ & $\checkmark$ & \\
\hline Bosea sp. 127 & Proteobacteria(1); Alphaproteobacteria(1); Rhizobiales(1); Bradyrhizobiaceae(1); Bosea(1); & & & & $\checkmark$ & $\checkmark$ & \\
\hline Bosea sp. 21B & Proteobacteria(1); Alphaproteobacteria(1); Rhizobiales(1); Bradyrhizobiaceae(1); Bosea(1); & & & & $\checkmark$ & $\checkmark$ & \\
\hline Bosea sp. 29B & Proteobacteria(1); Alphaproteobacteria(1); Rhizobiales(1); Bradyrhizobiaceae(1); Bosea(1); & & & & $\checkmark$ & $\checkmark$ & \\
\hline Bosea sp. 46 & Proteobacteria(1); Alphaproteobacteria(1); Rhizobiales(1); Bradyrhizobiaceae(1); Bosea(1); & & & & $\checkmark$ & $\checkmark$ & \\
\hline Bosea sp. 62 & Proteobacteria(1); Alphaproteobacteria(1); Rhizobiales(1); Bradyrhizobiaceae(1); Bosea(1); & & & & $\checkmark$ & $\checkmark$ & \\
\hline Bosea sp. 7B & Proteobacteria(1); Alphaproteobacteria(1); Rhizobiales(1); Bradyrhizobiaceae(1); Bosea(1); & & & & $\checkmark$ & $\checkmark$ & \\
\hline Hoeflea sp. HK425 & Proteobacteria(1); Alphaproteobacteria(1); Rhizobiales(1); Phyllobacteriaceae(1); Hoeflea(1); & & & & & $\checkmark$ & \\
\hline Rhizobium sp. SD404 & Proteobacteria(1); Alphaproteobacteria(1); Rhizobiales(1); Rhizobiaceae(0.96); Rhizobium(0.92); & $\checkmark$ & $\checkmark$ & $\checkmark$ & & & $\checkmark$ \\
\hline Roseovarius sp. EC-HK134 & Proteobacteria(1); Alphaproteobacteria(1); Rhodobacterales(1); Rhodobacteraceae(1); Roseovarius(1); & & & $\checkmark$ & $\checkmark$ & $\checkmark$ & $\checkmark$ \\
\hline Roseovarius sp. SD190 & Proteobacteria(1); Alphaproteobacteria(1); Rhodobacterales(1); Rhodobacteraceae(1); Roseovarius(1); & & & $\checkmark$ & $\checkmark$ & $\checkmark$ & $\checkmark$ \\
\hline Erythrobacter sp. HK427 & Proteobacteria(1); Alphaproteobacteria(1); Sphingomonadales(1); Erythrobacteraceae(1); Erythrobacter(1); & $\checkmark$ & & & & $\checkmark$ & \\
\hline Novosphingobium sp. $9 \mathrm{U}$ & Proteobacteria(1); Alphaproteobacteria(1); Sphingomonadales(1); Sphingomonadaceae(1); Novosphingobium(1); & $\checkmark$ & & & & $\checkmark$ & \\
\hline Sphingomonas sp. 8AM & Proteobacteria(1); Alphaproteobacteria(1); Sphingomonadales(1); Sphingomonadaceae(1); Sphingomonas(1); & & & & & $\checkmark$ & \\
\hline Sphingomonas sp. AX6 & Proteobacteria(1); Alphaproteobacteria(1); Sphingomonadales(1); Sphingomonadaceae(1); Sphingomonas(1); & & & & & $\checkmark$ & \\
\hline Sphingomonas sp. EC-HK361 & Proteobacteria(1); Alphaproteobacteria(1); Sphingomonadales(1); Sphingomonadaceae(1); Sphingomonas(1); & & & & & $\checkmark$ & \\
\hline Sphingomonas sp. EC-SD391 & Proteobacteria(1); Alphaproteobacteria(1); Sphingomonadales(1); Sphingomonadaceae(1); Sphingomonas(1); & & & $\checkmark$ & & $\checkmark$ & $\checkmark$ \\
\hline Sphingomonas sp. T1 & Proteobacteria(1); Alphaproteobacteria(1); Sphingomonadales(1); Sphingomonadaceae(1); Sphingomonas(1); & & & & & $\checkmark$ & $\checkmark$ \\
\hline Sphingorhabdus sp. 109 & Proteobacteria(1); Alphaproteobacteria(1); Sphingomonadales(1); Sphingomonadaceae(1); Sphingorhabdus(1); & & & & & $\checkmark$ & $\checkmark$ \\
\hline Burkholderia sp. $8 Y$ & Proteobacteria(1); Betaproteobacteria(1); Burkholderiales(1); Burkholderiaceae(1); Burkholderia(1); & & & $\checkmark$ & & & $\checkmark$ \\
\hline Burkholderiales bacterium $8 \mathrm{X}$ & Proteobacteria(1); Betaproteobacteria(1); Burkholderiales(1); Comamonadaceae(1); Variovorax(1); & & & $\checkmark$ & & $\checkmark$ & $\checkmark$ \\
\hline Massilia sp. 9I & Proteobacteria(1); Betaproteobacteria(1); Burkholderiales(1); Oxalobacteraceae(1); Massilia(1); & & & & & $\checkmark$ & \\
\hline Aeromonas sp. 8C & Proteobacteria(1); Gammaproteobacteria(1); Aeromonadales(1); Aeromonadaceae(1); Aeromonas(1); & $\checkmark$ & $\checkmark$ & & & $\checkmark$ & $\checkmark$ \\
\hline Aeromonas sp. 9A & Proteobacteria(1); Gammaproteobacteria(1); Aeromonadales(1); Aeromonadaceae(1); Aeromonas(1); & $\checkmark$ & $\checkmark$ & & $\checkmark$ & & $\checkmark$ \\
\hline Alteromonas sp. 154 & Proteobacteria(1); Gammaproteobacteria(1); Aeromonadales(1); Aeromonadaceae(1); Aeromonas(1); & & & & & $\checkmark$ & \\
\hline Alteromonas sp. 38 & Proteobacteria(1); Gammaproteobacteria(1); Alteromonadales(1); Alteromonadaceae(1); Alteromonas(1); & & & & & $\checkmark$ & \\
\hline Marinobacter sp. HK377 & Proteobacteria(1); Gammaproteobacteria(1); Alteromonadales(1); Alteromonadaceae(1); Marinobacter(1); & & & $\checkmark$ & $\checkmark$ & $\checkmark$ & $\checkmark$ \\
\hline Marinobacter sp. N1 & Proteobacteria(1); Gammaproteobacteria(1); Alteromonadales(1); Alteromonadaceae(1); Marinobacter(1); & & & & $\checkmark$ & $\checkmark$ & $\checkmark$ \\
\hline Pantoea sp. 111 & Proteobacteria(1); Gammaproteobacteria(1); Enterobacteriales(1); Enterobacteriaceae(1); Pantoea(1); & $\checkmark$ & & & & $\checkmark$ & \\
\hline Enterobacterales bacterium 8AC & Proteobacteria(1); Gammaproteobacteria(1); Enterobacteriales(1); Enterobacteriaceae(1); Yersinia(0.68); & & $\checkmark$ & & $\checkmark$ & $\checkmark$ & $\checkmark$ \\
\hline Halomonas sp. 113 & Proteobacteria(1); Gammaproteobacteria(1); Oceanospirillales(1); Halomonadaceae(1); Halomonas(1); & & $\checkmark$ & $\checkmark$ & $\checkmark$ & & $\checkmark$ \\
\hline Halomonas sp. 153 & Proteobacteria(1); Gammaproteobacteria(1); Oceanospirillales(1); Halomonadaceae(1); Halomonas(1); & & $\checkmark$ & $\checkmark$ & $\checkmark$ & & $\checkmark$ \\
\hline Halomonas sp. 156 & Proteobacteria(1); Gammaproteobacteria(1); Oceanospirillales(1); Halomonadaceae(1); Halomonas(1); & & $\checkmark$ & $\checkmark$ & $\checkmark$ & & $\checkmark$ \\
\hline Halomonas sp. 59 & Proteobacteria(1); Gammaproteobacteria(1); Oceanospirillales(1); Halomonadaceae(1); Halomonas(1); & & $\checkmark$ & $\checkmark$ & $\checkmark$ & & $\checkmark$ \\
\hline Halomonas sp. 98 & Proteobacteria(1); Gammaproteobacteria(1); Oceanospirillales(1); Halomonadaceae(1); Halomonas(1); & & $\checkmark$ & $\checkmark$ & $\checkmark$ & & $\checkmark$ \\
\hline Halomonas sp. $\mathrm{I3}$ & Proteobacteria(1); Gammaproteobacteria(1); Oceanospirillales(1); Halomonadaceae(1); Halomonas(1); & & $\checkmark$ & & $\checkmark$ & & \\
\hline Acinetobacter sp. 8BE & Proteobacteria(1); Gammaproteobacteria(1); Pseudomonadales(1); Moraxellaceae(1); Acinetobacter(1); & & $\checkmark$ & & & $\checkmark$ & \\
\hline Acinetobacter sp. 8I-beige & Proteobacteria(1); Gammaproteobacteria(1); Pseudomonadales(1); Moraxellaceae(1); Acinetobacter(1); & & & & & $\checkmark$ & $\checkmark$ \\
\hline Enhydrobacter sp. AX1 & Proteobacteria(1); Gammaproteobacteria(1); Pseudomonadales(1); Moraxellaceae(1); Enhydrobacter(0.85); & & & & & $\checkmark$ & \\
\hline Moraxellaceae bacterium 17A & Proteobacteria(1); Gammaproteobacteria(1); Pseudomonadales(1); Moraxellaceae(1); Enhydrobacter(0.91); & & & & $\checkmark$ & $\checkmark$ & \\
\hline Enhydrobacter sp. 8BJ & Proteobacteria(1); Gammaproteobacteria(1); Pseudomonadales(1); Moraxellaceae(1); Enhydrobacter(1); & & & $\checkmark$ & & $\checkmark$ & \\
\hline Pseudomonas sp. 8AS & Proteobacteria(1); Gammaproteobacteria(1); Pseudomonadales(1); Pseudomonadaceae(1); Pseudomonas(1); & & $\checkmark$ & & & $\checkmark$ & $\checkmark$ \\
\hline Pseudomonas sp. 8BK & Proteobacteria(1); Gammaproteobacteria(1); Pseudomonadales(1); Pseudomonadaceae(1); Pseudomonas(1); & & & & & $\checkmark$ & \\
\hline Pseudomonas sp. 80 & Proteobacteria(1); Gammaproteobacteria(1); Pseudomonadales(1); Pseudomonadaceae(1); Pseudomonas(1); & $\checkmark$ & & & & $\checkmark$ & \\
\hline Pseudomonas sp. $8 \mathrm{Z}$ & Proteobacteria(1); Gammaproteobacteria(1); Pseudomonadales(1); Pseudomonadaceae(1); Pseudomonas(1); & & & & $\checkmark$ & $\checkmark$ & $\checkmark$ \\
\hline Pseudomonas sp. 9Ag & Proteobacteria(1); Gammaproteobacteria(1); Pseudomonadales(1); Pseudomonadaceae(1); Pseudomonas(1); & & & & & & $\checkmark$ \\
\hline \multicolumn{2}{|c|}{$\begin{array}{l}\text { Number of compounds producible by the community; } \\
\text { Size of the minimal solution: }\end{array}$} & 506 & 484 & 549 & 492 & 504 & $\mathbf{5 9 0}$ \\
\hline
\end{tabular}


